\title{
Health Analysis of Footballer Using Big Data and Deep Learning
}

\author{
Tao Yang, ${ }^{1}$ Guoliang Yuan $\left(\mathbb{D},{ }^{2}\right.$ and Jing $\operatorname{Yan}^{3}$ \\ ${ }^{1}$ Institute of Physical Education, North Minzu University, Yinchuan, Ningxia 750021, China \\ ${ }^{2}$ College of Physical Education, HengShui University, Hengshui, Hebei 053000, China \\ ${ }^{3}$ Chinese Academy of Customs Administration, Qinhuangdao, Hebei 066004, China \\ Correspondence should be addressed to Guoliang Yuan; 601254@hsnc.edu.cn
}

Received 7 April 2021; Revised 30 April 2021; Accepted 24 May 2021; Published 16 June 2021

Academic Editor: Shah Nazir

Copyright (c) 2021 Tao Yang et al. This is an open access article distributed under the Creative Commons Attribution License, which permits unrestricted use, distribution, and reproduction in any medium, provided the original work is properly cited.

\begin{abstract}
With the development of information technology, health management and big data have risen and developed in recent years. Big data need proper analysis and shape in order to extract meaningful information from it. This paper analyzes the application status and prospects of big data in the field of health management. The results show that the most widely used big data in health management are intelligent wearable devices. Big data applications in football players' mental health monitoring systems and chronic disease health management systems also have a good prospect. The intelligent wearable device is applied to several aspects of sports work: teaching and sports training, real-time monitoring of football players' physical exercise process, collecting football players' heart rate, calorie consumption, exercise steps, and track, blood pressure, blood oxygen, and other physical exercise data; through monitoring the heart rate, we can get the intensity and duration of football players' physical exercise in school; through the calculation, we can also get the football players' time energy consumption and understand the overall situation of football players' physical exercise in school; through step counting and track monitoring, we can master the number of steps and track of football players; by monitoring the changes of blood oxygen and blood pressure of football players, we need to build a third-party residents' health information storage and analysis system and further realize the marketization of residents' health big data. The experimental results of the proposed study show the effectiveness of the proposed work.
\end{abstract}

\section{Introduction}

As people pay more attention to health problems, people pay more and more attention to health-related products, especially football players. With the social development and economic progress, more and more football players wear intelligent wearable devices. More and more football players use intelligent wearable devices to monitor their health problems. More and more people begin to pay attention to sports health management. With the development of the electronic communication industry, the smartphone industry has developed to an unprecedented peak. During this period, big smartphone manufacturers such as apple and Samsung encountered bottlenecks in mobile phone innovation. People put the development of smart devices into smart wearable devices, and various smart wearable devices have sprung up like mushrooms. In 2013, about 6.75 million smart wearable devices were sold in China, and it is expected to exceed 73.5 million in 2016 and develop into a huge market with a scale of 16.94 billion yuan [1] as intelligent wearable devices need to contact with the body and they have the advantage of detecting the body quality. Therefore, many manufacturers have launched intelligent wearable devices with fitness functions, and the development opportunities of intelligent wearable devices in the sports field will be unprecedented. As the intelligent wearable device is a new product of scientific and technological development, there is no unified conclusion on the definition of the intelligent wearable device [2].

In this paper, through the analysis of football players' personalized teaching, Sports Prescription Theory and attribution, cognitive dissonance, and behavior reinforcement theory, this paper studies and analyzes the football players in various countries. By using the research on the status quo of football players' physique and other aspects, this paper analyzes the optimization [3] effect of intelligent wearable 
equipment on the sports health management of football players. Also, through the support of society, government, enterprises, and the specific measurement of football players' physical condition, we can understand the various functions of wearable devices and analyze the different functions of different intelligent wearable devices. At present, the field of intelligent wearable devices is developing rapidly, and there are various kinds and quantities of intelligent wearable devices. Now for our football players, it is the priority to understand the real benefits that intelligent wearable devices can bring us. Secondly, we should make a targeted selection of devices and, finally, formulate a reasonable sports health plan to improve football games mobilize their physical quality to achieve the goal of sports health management. The following are the key contributions of the paper:

To analyze the application status and prospects of big data in the field of health management

To study big data applications in football players' mental health monitoring systems and chronic disease health management systems also have a good prospect

To apply intelligent wearable device for several aspects of sports work

To show the effectiveness of the proposed study through experimental results

This paper is organized as follows: Section 2 presents the research overview of the intelligent wearable devices. Section 3 is the representation of the design and implementation of a wearable human motion capture system. The details of design of the motion capture hardware platform are given in Section 4. Section 5 briefly represents the report on the total amount of sexual activity and calorie consumption of football players. Section 6 represents the details of classification and function of intelligent wearable devices. The value of intelligent wearable devices in sports activities is given in Section 7. This paper is concluded in Section 8.

\section{A Research Overview of Intelligent Wearable Devices}

The Baidu Encyclopedia defines intelligent wearable devices as "wearable intelligent devices" as the general term of wearable devices, such as glasses, gloves, watches, clothing, and shoes, by applying wearable technology to intelligently design and develop wearable devices. Li Yang believes that "wearable devices refer to intelligent devices with the independent operating system, system application, upgrading and scalability, worn by the human body, and realizing continuous interaction." This paper agrees with Li Yang's statement that wearable devices refer to intelligent devices that adopt the independent operating system, have system applications, upgrade and expansion, are worn by the human body, and realize continuous interaction [4]. The research on intelligent wearable devices is less, more just as science and technology news reports, domestic and foreign research on intelligent wearable devices in the field of sports is less, so this study has a certain degree of innovation for the research of intelligent devices in the field of sports to provide a reference and reference.

\subsection{Development and Evolution of Intelligent Wearable} Devices. Early wearable devices can be traced back to the $13^{\text {th }}$ century, when the first pair of glasses with crystal lenses appeared. In China, in the 17 th century, the abacus ring, the rudiment of intelligent wearable devices, appeared. In 1762, John Harrison invented the pocket watch. In 1907, the first watch was born. People began the watch era. In 1977, HP developed the HP 01 algebra calculator watch, which sold for $\$ 600$. Computer shoes were designed in 1978 to predict roulette results. In 1993, BBN completed the Pathfinder system. The system is a wearable computer equipped with GPS and a radiation detection system. In 1994, with the advent of wearable cameras, Steve Mann developed a "wearable wireless camera." This device can transmit pointto-point through amateur TV frequency, and images can be transmitted from head-on analog cameras to SGI base stations. In 1998, Seiko launched a smartwatch rupture compatible with Windows 95. In 2010, Nike launched the Nike + SportBand device, one of the founders of smart wearable devices, which can "communicate" with sensors hidden in sneakers and let users know more about their running. In April 2012, Google released Google glasses, bringing intelligent wearable devices into public view. In 2013, Samsung launched the Samsung gear smartwatch. In 2015, Apple launched the Apple Watch smartwatch, and then, 2015 was called the first year of smart wearable devices.

\section{Design and Implementation of a Wearable Human Motion Capture System}

Motion capture technology is to obtain 3D motion information of the human body in real-time by computer vision [5-7] technology and then analyze and use the information. Fleischer invented dynamic mask technology in 1915, which is regarded as the ancestor of motion capture technology. In the 1970s, the Computer Graphics Laboratory of New York designed an optical motion capture device, which can display actors' actions on the screen in real time, which has become the beginning of modern motion capture technology. Modern motion capture systems are based on different capture principles, including acoustic, electromagnetic, optical, mechanical, video capture, and inertial. The accuracy of acoustic and electromagnetic capture systems is relatively poor, and it is affected by environmental noise and magnetic fields. An optical capture system has high precision, but it is complex to install, costly, and has high environmental requirements. The mechanical motion system is difficult to wear and will affect the free activity of the human body. Although the video capture system does not need to wear sensor nodes on the human body, its algorithm is complex, and it is difficult to realize. The motion capture system based on an inertial sensor has the characteristics of portable wear, simple operation, and low cost. It can continuously collect real-time motion information of all parts of the human body without the limitation of time and place. It has been widely 
used in fall monitoring, sports training, military training, somatosensory games, virtual reality, and augmented reality. For example, in the aspect of fall monitoring, through wearing inertial sensors on the wrist or other parts, real-time acquisition of the acceleration, angular velocity, and other information of the wearing part, when the human body suddenly falls, the inertial data of the wearing part will change suddenly, to judge that the human body falls and timely ask the family members and medical staff for help.

To capture human motion in real time, a wearable human motion capture system is designed. Each IMU is composed of a micro-MEMS 3-axis gyroscope, MEMS 3-axis accelerometer, MEMS 3-axis magnetometer, and MCU. An MCU obtains the data of each sensor and calculates the attitude angle of corresponding parts by using the extended Kalman filter based on quaternion. The data are uploaded to the computer in real-time through the CAN bus and Bluetooth module. The computer drives the virtual human body movement through $\mathrm{VC}++$ and OpenGL program to realize real-time human motion reproduction.

\subsection{The Overall Design of the System Software Platform.} The human motion capture system is composed of a lower computer and an upper computer. The lower computer is distributed in the whole body of sensor nodes, real-time sensor node data, through the wireless module, and sent to the PC. The upper computer vision [8-10] platform runs on the PC. The PC receives the data of sensor nodes in real-time through the wireless antenna. The software platform analyzes and processes these data and reconstructs the calculated human posture into computer graphics in real time. To make the software platform achieve certain functions and usability, it is combined with the requirements of the motion capture system itself.

3.1.1. Serial Data Receiving. It is mainly responsible for realtime monitoring of the wireless data received in the serial port of the computer [11], receiving the data distributed in each key sensor node of the human body in real time, distinguishing the nodes of each sensor data, and sending these data to the data preprocessing module.

3.1.2. Data Preprocessing. It mainly completes the preprocessing process of the original signal discussed in Section 3 . The signal with error and noise is compensated and filtered. The data are divided into two parts: one part is output to the attitude calculation module. The other part saves the data in real time to facilitate other related simulation experiments.

3.1.3. Loading and Driving of the Human Model. This is in charge of importing the human model made in Section 4 into the $3 \mathrm{D}$ scene of the software and driving the human body model in real time by using the data processing module to solve the human posture.
3.1.4. Software Platform Appearance Beautification. This part of the function is mainly responsible for the visual control, title bar, title, and menu bar interface beautification work. In the $\mathrm{VC}++$ development environment, there is no corresponding beautification interface for visual control, menu bar, and toolbar, which needs to be customized and processed.

3.2. Detailed Design of the Software Platform. The system uses wireless to the serial port to complete the communication between the upper computer and the lower computer. In $\mathrm{VC}++$, the serial port needs to be monitored in real time to complete the data receiving. In common $\mathrm{VC}++$, the methods of receiving serial data include calling system API directly, third-party serial port class, and COM-based serial port control. Serial port control has good performance, shielding some complex bottom operations, stable performance, and shortening the software development cycle. This software platform uses the ActiveX control MSComm developed by Microsoft. McComb control supports both the active query mode and event-driven model. In the process of serial port initialization, firstly, the serial port control is initialized, including setting serial port number, baud rate, and input and output buffer space size. Finally, the buffer data are read in advance once, and the data remaining in the buffer are cleared. To prevent data deposition and loss, the data receiving part is encapsulated into a separate module. At the same time, the software improves the concurrency and opens up new threads in the module. In $\mathrm{VC}++$, each thread is inherited from a twin thread class. There are two types of threads, one is a worker thread without a message queue, and the other is a user interface thread with a message queue and the maintenance message loop mechanism. User interface threads are mainly used to interact with users, respond to peripheral input in real-time and process events triggered by the operating system. The worker thread does not need to interact with the user and is usually used to deal with tasks with a large amount of computation, such as printing service and transmission. The data receiving module does not need to interact with the user, it only needs to ensure the safe and stable data receiving, so it is more appropriate to use worker thread for receiving thread.

The format of the data frame is sent by the sensor node. When reading the serial buffer data, the original data of the sensor node in the frame are analyzed. On the one hand, the data are transferred to the attitude calculation module; on the other hand, the original data are saved as a file in a certain format, which is convenient for further analysis and research of sensor node data offline. Generally, the offline data collected are mainly used in algorithm research, which must be stored according to certain rules, and the file format is the general format. At the same time, the software platform saves the original data collected by the gyroscope, velocimeter, and magnetometer to the subfolder under the software path. To read the data conveniently, the data are saved as an XLS file.

$\mathrm{VC}++$ is selected as the software development environment, and the MFC framework and DirectX graphical 
interface library are used to complete the loading and driving of the human body model. A multifunctional application platform integrating data receiving and storage, real-time attitude calculation, and real-time human motion reconstruction is completed. The software of human motion and posture capture system is designed, and the functions of real-time motion capture and three-dimensional structure are completed.

\section{Design of the Motion Capture Hardware Platform}

According to the principle of inertial motion capture equipment and current technology, generally, for singleperson motion capture, the model shown in Figure 1 can be used to describe. For the model, users wear 11 inertial motion sensors, which are distributed in the left upper arm, left forearm, right big arm, right forearm, left thigh, left leg, right thigh, right leg, head, chest, and waist. Each motion sensor outputs acceleration, magnetic field strength, and angular velocity inertial parameters. The sensors are connected by a CAN bus, which can supply power to the sensors with high data rate and reliability. The concentrator is the bridge between the sensor and the computer. The sensor node first sends the data to the concentrator and then forwards it to the receiver. The receiver transmits the data to the computer through a USB port. The concentrator and sensor are connected by a wire, and wireless communication is adopted between concentrators and computers.

4.1. The Part of the Motion Sensor. The functions of the motion sensor include data acquisition, attitude angle settlement, and wired communication for three different types of inertial devices. The block diagram of the hardware system is shown in Figure 2. As can be seen from Figure 2, the motion sensor mainly includes five parts: an MCU, acceleration sensor, gyroscope sensor, magnetometer, and CAN interface. The MCU consists of an asynchronous serial communication bus (I2C), asynchronous receiver transmitter (UART), keys, and other modules. It controls a series of operations of nodes. The nodes are powered by wire, and the MCU controls the data acquisition, attitude angle calculation, and CAN transceiver of the three sensors.

The main chips used in motion sensors include STM32F103 (MCU) of St, mpu6050 (3-axis acceleration juice and 3-axis gyroscope) of InvenSense, hmc5883 I (3-axis magnetometer) of honeywe11, and sn65hv D230 (can transceiver) of Ti. The parameters of each chip are shown in Table 1.

\section{Report on the Total Amount of Sexual Activity and Calorie Consumption of Football Players}

The specific calculation of football players' sports energy consumption is also an important way to understand the number of football players' activity calories are an important reference index to calculate human energy consumption. There are many ways to calculate the energy consumption of
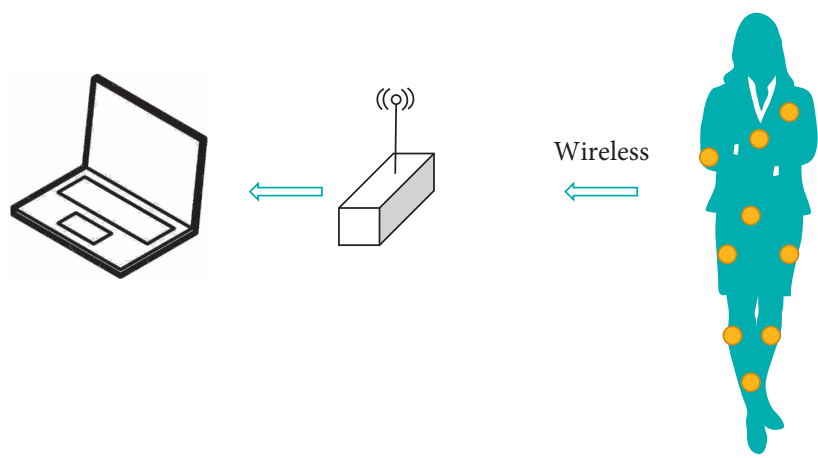

Figure 1: Motion system model.

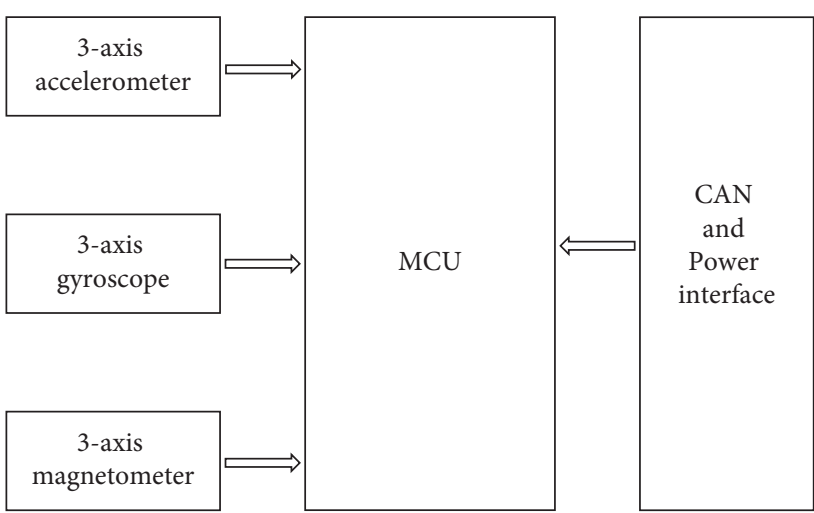

FIGURE 2: Block diagram of the motion sensor system.

TABLe 1: Chip parameters.

\begin{tabular}{lcc}
\hline Type & Name & Parameter \\
\hline Accelerometer & MPU6050 & $\pm 250 / 500 / 1000 / 2000^{\circ} \mathrm{sec}$ \\
Gyroscope & MPU6050 & $\pm 2 / 4 / 8 / 16 \mathrm{~g}$ \\
Magnetometer & HMC5883 L & \pm 8 gauess \\
MCU & STM32F103 & $90 \mathrm{DMIPS}$ \\
CAN & SN65HVD230 & $1 \mathrm{Mb} / \mathrm{s}$ \\
\hline
\end{tabular}

football players in stage activities. At present, there are mainly the direct heat measurement methods, indirect side heat method, double standard water detection method, heart rate detection method, pedometer detection method, and triaxial acceleration sensor detection method [12]. Each detection method has its advantages and disadvantages. At present, the commonly used energy monitoring methods in intelligent wearable devices are step counting monitoring, heart rate monitoring, and triaxial acceleration sensor monitoring. Step counting monitoring mainly records the number of human movement steps and then estimates the energy consumption of the human body. Because in some unconventional running and walking movements, the energy consumption of the human body is not only manifested as the distance between the number of steps and the moving position, there is a big error in calculating the energy consumption of football players by using the step counting principle [13];heart rate detection mainly measures the energy consumption of human body according to the change of human heart rate. Heart rate can effectively judge the 
intensity of human exercise and oxygen consumption. According to the corresponding intensity to measure the energy consumption of football players, the equipment requirements are simple, and the accuracy rate of heart rate measurement is also high. However, heart rate is easily affected by the external environment and internal differences of testers, which leads to detection. There is an error. Meijer and other related researchers have also found that the measurement of human energy consumption through heart rate, step counting, three-dimensional acceleration, and other related principles is the ideal way to measure the energy consumption of the human body [14]. The calculation and construction of energy consumption based on the characteristic value GM is one of them. GM value is the geometric average value of three-axis acceleration data as the characteristic quantity to calculate the energy consumption of human body movement:

$$
G M=\sqrt{\frac{\left(A_{x}^{2}+A_{y}^{2}+A_{z}^{2}\right)}{3} .}
$$

$A_{x}, A_{y}$, and $A_{z}$ are the output values of the acceleration sensor in the $X-, Y$-, and $Z$-axis, respectively, and the unit is g. After studying the correlation between the geometric mean of the three-axis acceleration data and the energy consumption of the human body, Yang Huiliang proposed a calculation equation of human energy consumption based on the eigenvalue $G M$.

$$
E F=\frac{\left((1 / 2) u^{\bullet} m g^{\bullet} \int_{a 1}^{a 2} \mathrm{~d} a \int_{t 1}^{t 2} t \mathrm{dt}\right)}{4.18} .
$$

\section{Classification and Function of Intelligent Wearable Devices}

There are various types of intelligent wearable devices. "From the perspective of the products used in the wearable intelligent devices, $30 \%$ of the products are worn on the wrist and $26 \%$ and $22 \%$ of the products are worn on the upper body and head, respectively. $15 \%$ of the products do not have clear requirements for the wearing parts. However, the feet seem to be the least favored part of the intelligent wearable devices, and only $7 \%$ of the products choose the feet." [13].

A fan distribution map of intelligent wearable devices is classified by function. From this, we can see that the current wearable devices are mainly used in the field of medical health and sports fitness. Using wearable devices that can monitor ECG signals to monitor the heart condition of users in walking, running, and other activities can prevent heart disease. A wearable sensor is used to detect the change of heart rate and the number of walking steps during exercise and calculate the heat and calories consumed by the user, evaluate the health level of the user, and formulate a reasonable fitness plan for the user.

According to the wearing position and the temperature monitoring circuit function module, the wearable device is subdivided according to the position, and the wearable device is classified into the following categories according to the functional classification. Wearable devices can be electronic products worn on the head, neck, arm, wrist, or finger or embedded in clothes or shoes. As can be seen from the figure, wearable devices are mainly worn at the wrist. It is because people are used to wearing bracelets or watches, and the wearable devices on the wrist are portable, consume low power, and small in size.

\section{The Value of Intelligent Wearable Devices in Sports Activities}

According to Fjord's research and analysis of 27 well-known wearable intelligent devices, "about $70 \%$ of the main functions of these products are used in sports and health, $23 \%$ is used in social communication, and the remaining $7 \%$ is used for monitoring and improving sleep." In all the listed intelligent wearable devices, almost all intelligent wearable devices have the same function, that is, the collection and collation of human motion data, which shows that intelligent wearable [15] devices have great development value in the field of sports.

At present, there are various types of intelligent products in the market, including medical and healthcare products, intelligent rings, intelligent headbands, intelligent bracelets, intelligent glasses, smartwatches, and intelligent antiloss devices. Easy operation is the basic requirement of intelligent equipment, and intelligence is its development trend. According to the application functions of the equipment, it is divided into the following five categories.

The first category is life sports health equipment. This kind of equipment is mainly used to collect human movement data, physical fitness health data, and life work and rest data. The main form of the product is the bracelet. The main functions of the products are heart rate monitoring, exercise measurement, blood oxygen measurement, sedentary reminder, sleep quality monitoring, exercise mode, body surface temperature monitoring, and calorie consumption. For example, obese people can use the device's exercise record and calorie consumption calculation function to make a practical exercise plan for themselves [16]. Under the supervision of the equipment, they can control the exercise heart rate, achieve their own exercise goals, and improve the efficiency of physical exercise. This kind of equipment can help people better understand their physical conditions, find their body abnormalities in time, and track the change process of various indicators of the body. In the current environment where people pay more and more attention to their health problems, life sports health products are an important part of the intelligent wear market.

The second category is leisure and entertainment equipment, mainly to meet people's daily leisure and entertainment needs; the main forms of products are watches and glasses. For example, Google glasses can get information notification and remind in time to avoid missing important information; at the same time, it supports voice input, so that you can process the important information received in time, without affecting the use of some mobile phones when it is inconvenient. Also, Google glasses have a convenient phototaking function, which can help people capture beautiful 
moments in time. Another example is VR intelligent virtual glasses, with a high-definition display function, which can make game pictures and movie pictures more realistic, enhance people's experience, and 3D vector sound field effect, which makes people feel like they are in the scene [17]. There are also smartwatch products and smartwatches with extended functions of mobile phones, voice navigation, message push, call reminder, and intelligent alarm clock functions so that people's lives are more convenient.

The third category is safety protection equipment, which mainly meets the needs of the elderly and children. Children's products are mainly reflected in children's watch products. Guardians can remotely monitor children's phone calls. Through the GPS positioning system, the specific location of children can be known, and the family number can be set. When necessary, children can be contacted in time, and communication can be joined through control record friends and refuse strangers to add, to further ensure the safety of children's external environment. Compared with the mobile phone, the telephone watch also plays a communication function, but it is more convenient for children to use. At the same time, the smartphone watch also has the function of an alarm when the watch falls off, which makes the phone watch a real tool for parents to find their children in time. The safety products for the elderly mainly use the GPS positioning function to let the family know the whereabouts of the elderly in time to prevent loss and accidents.

The fourth category of products is medical and healthcare equipment, mainly to meet the needs of specific groups of people to monitor their health indicators [18]. For example, the household finger clip measuring instrument is equivalent to a mobile "physical examination doctor." It can detect the basic health indicators of the human body at any time, including heart rate, blood oxygen, blood pressure, respiratory rate, $\mathrm{PI}$, and other indicators, which can be automatically detected in 60 seconds, upload data records, generate physical examination reports, and provide analysis suggestions and is a health housekeeper. The data recording function of the device can generate weekly reports, monthly reports, and annual reports, which is convenient for users to know their past health status at any time.

The fifth category is smart home products, which mainly meet the control needs of household appliances and furniture in daily life [19-23]. Through WiFi or other wireless ways, the control of household appliances such as the water heater, TV, and air conditioner can be realized to save electricity and avoid fire accidents. At the same time, HD equipment can be used to intelligently monitor the situation of the home; for example, there are nannies taking care of children in the family, worrying about the health of the elderly, worrying about the pets in the family, and worrying about theft of property in the family by thieves. Effective protective measures can be taken for all of these through the intelligent device. Through the use of smart home products, people can enjoy home life more intelligently.

Supervising the movement and adjusting the movement state: intelligent wearable devices can transmit real-time motion parameters to users, such as heartbeat, blood pressure, and energy consumption. The current intelligent wearable devices can monitor the user's activity parameters and timely transmit the real-time motion parameters of users to the intelligent wearable devices. Therefore, during aerobic exercise such as running, swimming, and cycling, users can monitor their heart rate, blood pressure, and other information at any time. In the process of sports, according to these parameters, we can adjust our own sports situation so that the sport's participants can adjust their state at any time and control their sports.

7.1. Correct Movement and Efficient Training. Recently, some companies have made deeper development in the field of wearable technology. The wearable devices of LUMO LFT and Wa Hoo can capture various movements of the arms and legs. By using artificial intelligence algorithms and sensors that can measure the motion conditions in 3D space, we can compare the body posture of users in swimming, running, or archery with the motion data collected from professional athletes by ideal motion models. Therefore, for the beginners of sports, comparing these data parameters can show how to regulate their actions, and the learning process of sports can also be more scientific and efficient [24-27].

7.2. Prevention of Sports Injury of Athletes. The intelligent wearable device can transmit the athlete's body data to the medical and healthcare personnel in real time. According to the athlete's physical condition, the sports training is specially customized, and the athlete's fatigue condition is analyzed and modeled to understand the rise and fall of the athlete's competitive state in real time. Based on this, the athletes can maintain their physical condition in the best competitive state. Through the collection of athletes' sports data, big data can be used for processing. Athletes can also collect sports data through wearable devices in the usual competition. The data modeling can be carried out for the physical condition data of athletes before the injury so that medical and healthcare personnel can track the physical condition of athletes in real time, and early warning can be timely carried out before athletes are injured, to reduce sports injury.

7.3. Improving the Competitive Level of Athletes. The future is an "Internet of Things era"; that is, people can control the automobile, electrical appliances, and other equipment through intelligent devices. In the field of sports, intelligent wearable devices can be designed intelligently with the equipment used by athletes and the equipment of athletes. When athletes use competition equipment, data collection equipment is added to the equipment. At the same time, it is combined with the intelligent wearable equipment of athletes, so that the sports mobilization can understand the advantages and disadvantages of their actions more correctly and the athletes and competition equipment can be realized. Now, "character interconnection," for example, ballplayers can correct their actions according to the feedback of sensors 
on the football and the hitting point information, is used to continuously improve their competitive level. At the same time, the intelligent glasses in the intelligent wearable device can reproduce the virtual scene and help the athletes better integrate into the atmosphere of the competition. "The NFL (National Football League) has planned to introduce intelligent wearable technology to the NFL arena, and NFL players are using wearable devices to prepare for their professional sports careers." With the development of science and technology, intelligent wearable devices can be more and more refined, recording sports data without the athletes' awareness, and constantly improve the competitive level of athletes.

\section{Conclusions}

With Apple's release of the Apple watch, 2015 is known as the first year of intelligent wearable devices. The era of intelligent wearable devices has come, and the impact on sports will be far reaching. However, the development time of intelligent wearable devices is relatively short, and there are still many problems to be solved, such as endurance, comfort, data acquisition, and other issues, which are still an important factor restricting the development of intelligent wearable devices. In all aspects of sports, intelligent wearable devices can be perfectly combined with it, and the development of intelligent wearable devices can better promote the development of sports, making sports more scientific, efficient, and easy to learn. Intelligent wearable devices have begun to penetrate all aspects of sports. Although intelligent wearable devices have just entered the initial stage of development, every scientific and technological progress requires constant attempts. The proposed study analyzes the application status and prospects of big data in the field of health management. To study big data applications in football players' mental health, monitoring systems and chronic disease health management systems that also have a good prospect are presented in this study. The application of an intelligent wearable device for several aspects of sports work is part of this study. Therefore, with the continuous development of science and technology, in the future, intelligent wearable devices will occupy an important position in the field of sports. The effectiveness of the proposed study is shown through experimental results.

\section{Data Availability}

The data used to support the findings of this study are included within the article.

\section{Conflicts of Interest}

The authors declare that they have not conflicts of interest regarding the publication of this paper.

\section{Acknowledgments}

This work was supported by "the Fundamental Research Funds for the Central Universities," North Minzu University (2019XYSTY01).

\section{References}

[1] M. Jiang, Thoughts and Suggestions on the Development Prospect of Intelligent Wearable Devices Mobile Communication, Springer, Berlin, Germany, 2014.

[2] L. I. U. Feng, H. A. N. Jing-Long, Q. I. Ji, Y. U. Jia-Luo, L. I. Wen-Peng, and L. I. Bo-Wei, "Research and application progress of intelligent wearable devices," Chinese Journal of Analytical Chemistry, vol. 49, no. 2, pp. 159-171, 2021.

[3] Y. Li and J. Cao, "WSN node optimal deployment algorithm based on adaptive binary particle swarm optimization," ASP Transactions on Internet of Things, vol. 1, no. 1, pp. 1-8, 2021.

[4] M. Chen, Y. Ma, Y. Li, D. Wu, Y. Zhang, and C.-H. Youn, "Wearable 2.0: enabling human-cloud integration in next generation healthcare systems," IEEE Communications Magazine, vol. 55, no. 1, pp. 54-61, 2017.

[5] J. Zhang, J. Sun, J. Wang, and X.-G. Yue, "Visual object tracking based on residual network and cascaded correlation filters," Journal of Ambient Intelligence and Humanized Computing, vol. 20, 2020.

[6] R. Liu, X. Ning, W. Cai, and G. Li, "Multiscale dense crossattention mechanism with covariance pooling for hyperspectral image scene classification," Mobile Information Systems, vol. 14, 2021.

[7] X. Ning, F. Nan, S. Xu, L. Yu, and L. Zhang, "Multi-view frontal face image generation: a survey. concurrency and computation," Practice and Experience, vol. 24, Article ID e6147, 2020.

[8] W. Cai, Z. Wei, R. Liu, Y. Zhuang, Y. Wang, and X. Ning, "Remote sensing image recognition based on multi-attention residual fusion networks," ASP Transactions on Pattern Recognition and Intelligent Systems, vol. 1, no. 1, pp. 1-8, 2021.

[9] X. Ning, Y. Wang, W. Tian, L. Liu, and W. Cai, "A biomimetic covering learning method based on principle of homology continuity," ASP Transactions on Pattern Recognition and Intelligent Systems, vol. 11, no. 1, pp. 9-16, 2021.

[10] Z. Huang, Y. Zhang, Q. Li et al., "Joint analysis and weighted synthesis sparsity priors for simultaneous denoising and destriping optical remote sensing images," IEEE Transactions on Geoscience and Remote Sensing, vol. 58, no. 10, pp. 6958-6982, 2020.

[11] Q. Liu, L. Cheng, A. L. Jia, and C. Liu, "Deep reinforcement learning for communication flow control in wireless mesh networks," IEEE Network, vol. 35, no. 2, pp. 112-119, 2021.

[12] B. Su, Design and Implementation of Human Posture Monitoring System Based on Acceleration Sensor, Northeast University, Boston, MA, USA, 2011.

[13] F. Shuntian, "Development status and trend of wearable devices," Research and development, vol. 53, no. 3, 2014.

[14] H. Li, "Discussion on the application prospect of wearable devices in the field of leisure sports," 2015.

[15] J. Deng, H. Liu, X. Yang, and Q. Cheng, "Current situation and future development trend of wearable intelligent devices," 2016.

[16] Q. Han, "Some thoughts on promoting the application of big data under the new situation," Scientific and Technological Innovation and Application, vol. 9, 2017.

[17] S. Zhao and X. Xu, "The application of wearable devices in Physical Education Teaching -- an analysis based on the SWOT model," Contemporary Sports Science and Technology, vol. 6, no. 34, pp. 111-112, 2016.

[18] G. Tang and H. Qin, "Min. Extension of Internet plus to physical education -- Application of smart wearable 
equipment in school physical education," Sports Technology, vol. 37, no. 3, pp. 143-144+150, 2016.

[19] Z. He, Design and Implementation of the Human Energy Consumption Detection System Based on the Acceleration Sensor, Harbin Institute of Technology, Harbin, China, 2016.

[20] B. Yang, Analysis of the Current Situation and Future Development Trend of Wearable Devices Wuhan Decision Information Research and Development Center, Decision, and Information magazine, School of economics and management, Peking University, Beijing, China, 2015.

[21] J. Deng, H. Liu, X. Yang, and Q. Cheng, "Current situation and future development trend of wearable intelligent devices," Heilongjiang Science and Technology Information, vol. 28, p. $135,2015$.

[22] Y. Zhao and T. Zhang, "Current situation analysis of intelligent wear in the health field [small," Chinese Journal of Health Information Management, vol. 12, no. 4, pp. 354-358, 2015.

[23] B. Li, X. Yu, X. Yang, and Y. Zheng, "Wearable exercise intensity monitoring system," Computer System Application, vol. 24, no. 5, pp. 32-39, 2015.

[24] C. Wang, Design and Implementation of Wearable MultiSensor Human Daily Activity Monitoring System, Nanjing University of Technology, Nanjing, China, 2015.

[25] P. Wang, "Fitness monitoring is an important market for wearable devices," Import and Export Manager, vol. 10, pp. 46-47, 2014.

[26] Y. Zhuang, "Sports and health products lead to the development of the wearable device market," Electronics World, vol. 21, no. 6, pp. 3-6, 2014.

[27] Y. Geng, Hui, L. Yang, and H. Jiang, "Development status and prospect of wearable devices," Electronic Science and Technology, vol. 10, no. 2, pp. 238-245, 2014. 